Pacific Journal of Mathematics

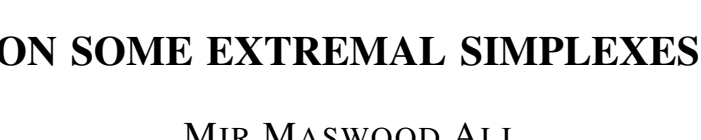




\title{
ON SOME EXTREMAL SIMPLEXES
}

\author{
MIR M. ALI
}

Let $A$ be a fixed point in $n$-dimensional Euclidean space. Let $B_{1}, B_{2}, \cdots, B_{n+1}$ be the vertices of a simplex $S_{n}$ of $n$ dimensions, that is, the $n+1$ vertices do not lie on a $(n-1)$ dimensional subspace. Let $d_{i}$, assumed to be positive, be the distance of $B_{i}$ from $A$, and let $l_{i j}$ be the cosine of the angle between the straight lines $A B_{i}$ and $A B_{j}$ for $i, j=1,2, \cdots$, $n+1$. Let $\pi_{i}$ denote the $(n-1)$-dimensional hyperplane passing through all the vertices of $S_{n}$ except $B_{i}$, let $p_{i}$, assumed positive, be the perpendicular distance of $\pi_{i}$ from $A$, and let $m_{i j}$ denote the cosine of the angle between the normals from $A$ to $\pi_{i}$ and $\pi_{j}$ for $i, j=1,2, \cdots, n+1$. The present paper deals with the following problems.

(a) An expression for the content of $S_{n}, C\left(S_{n}\right)$ say, in terms of $d_{i}$ and $l_{i j}$ for $i, j=1,2, \cdots, n+1$ is first obtained. Then leaving $d_{1}, d_{2}, \cdots, d_{n+1}$ fixed, values of $l_{i j}$, say $l_{i j}^{*}$, are determined in such a manner that $C\left(S_{n}\right)$ is a maximum, and the maximum value of $C\left(S_{n}\right)$ is obtained for the two cases that arise: (i) when $A$ is inside $S_{n}$, (ii) when $A$ is outside $S_{n}$. The latter case does not arise when $d_{1}=d_{2}=\cdots=d_{n+1}$.

(b) An expression for $C\left(S_{n}\right)$ is obtained in terms of $p_{i}$ and $m_{i j}, i, j=1,2, \cdots, n+1$. Then leaving $p_{1}, p_{2}, \cdots, p_{n+1}$ fixed, values for $m_{i j}$, say $m_{i j}^{*}$, are determined in such a manner that $C\left(S_{n}\right)$ is a minimum, and such $C\left(S_{n}\right)$ is computed for the two cases that arise depending on (i) whether $A$ is inside $S_{n}$ or (ii) $A$ is outside $S_{n}$. The latter case does not arise when

$$
p_{1}=p_{2}=\cdots=p_{n+1} \text {. }
$$

The results are stated below.

(a) The content of $S_{n}, \max C\left(S_{n}\right)$ and $l_{i j}^{*}$ are given by

$$
\begin{gathered}
n ! C\left(S_{n}\right)=\left|\left(l_{i j} d_{i} d_{j}+1\right)\right|^{1 / 2} \\
\max \left(n ! C\left(S_{n}\right)\right)^{2}=-u^{-1} \prod_{i=1}^{n+1}\left(d_{i}^{2}-u\right) \\
l_{i j}^{*}=u /\left(d_{i} d_{j}\right) \text { for } i, j=1,2, \cdots, n+1 ; i \neq j,
\end{gathered}
$$

where $u$ satisfies the equation

$$
1+u \sum_{i=1}^{n+2}\left(d_{i}^{2}-u\right)^{-1}=0 .
$$

The unique negative root for $u$ in (1.4) corresponds to the case when $A$ is inside $S_{n}$. When the relation

$$
d_{1}=d_{2}=\cdots=d_{n+1}
$$

is not satisfied, the smallest positive root for $u$ in (1.4) corresponds to the case when $A$ is outside $S_{n}$. Other roots for $u$ in (1.4), if any, are inadmissible. 
(b) The content $C\left(S_{n}\right)$, min $\left(C\left(S_{n}\right)\right)$ and $m_{i j}^{*}$ are given by

$$
\left(n ! C\left(S_{n}\right)\right)^{2}=\left|\left(p_{i} p_{j}+m_{i j}\right)\right|^{n} / \prod_{i=1}^{n+1}\left|M_{i i}\right|
$$

where $\left|M_{i i}\right|$ is the cofactor of $m_{i i}$ in $\left|\left(m_{i j}\right)\right|$ and

$$
\min \left(n ! C\left(S_{n}\right)\right)^{2}=-v^{-1} n^{2 n} \prod_{i=1}^{n+1}\left(p_{i}^{?}-v\right)
$$

and

$$
m_{i j}^{*}=v /\left(p_{i} p_{j}\right) \text { for } i \neq j ; i, j=1,2, \cdots, n+1 ;
$$

where $v$ satisfies the equation

$$
1+v \sum_{i=1}^{n+1}\left(p_{i}^{2}-v\right)^{-1}=0 .
$$

The unique negative root for $v$ in $(1.8)$ corresponds to the case when $A$ is inside $S_{n}$. When the relation

$$
p_{1}=p_{2}=\cdots=p_{n+1}
$$

is not satisfied, the smallest positive root for $v$ in (1.8) corresponds to the case when $A$ is outside $S_{n}$. All other roots, if any, are inadmissible.

When $d_{1}=d_{2}=\cdots=d_{n+1}$, we obtain the special result that the largest simplex inscribed in a sphere of $n$-dimensions is a regular one, while when $p_{1}=p_{2}=\cdots=p_{n+1}$ the smallest simplex circumscribing a sphere is a regular one.

The coordinates of $B_{i}$ referred to a $n$-dimensional Cartesian coordinate system with origin at $A$ will be denoted by $\left(x_{i, 1}, x_{i, 2}, \cdots, x_{i, n}\right)$. $\left(x_{1}, x_{2}, \cdots, x_{n}\right)$ will denote a general point in the $n$-space.

2. Extremal simplex determined by the distance of vertices. The content of $S_{n}$ is given by (Sommerville, p. 124) $n ! C\left(S_{n}\right)=|V|$ where

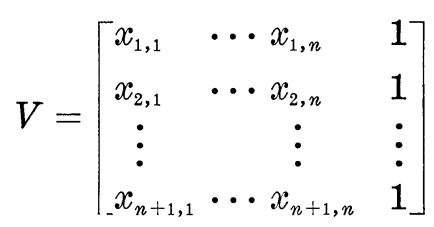

so that $\left(n ! C\left(S_{n}\right)\right)^{2}=\left|V V^{\prime}\right|=\left|\left(w_{i j}\right)\right|$ say, where

$$
\begin{aligned}
w_{i j} & =1+s_{i j} \text { for } i, j=1,2, \cdots, n+1 ; \text { and } \\
\left(s_{i j}\right) & =\left[\begin{array}{ccc}
x_{1,1} & \cdots & x_{1, n} \\
x_{2,1} & \cdots & x_{2, n} \\
\vdots & & \vdots \\
x_{n+1,1} & \cdots & x_{n+1, n}
\end{array}\right]\left[\begin{array}{ccc}
x_{1,1} & \cdots & x_{1, n} \\
x_{2,1} & \cdots & x_{2, n} \\
\vdots & & \vdots \\
x_{n+1,1} & \cdots & v_{n+1, n}
\end{array}\right]^{\prime} \\
& =\left(l_{i j} d_{i} d_{j}\right) .
\end{aligned}
$$


Hence we have proved (1.1).

We note that $s_{i i}=d_{i}^{2}$, for $i=1,2, \cdots, n+1$. From (2.3) we also note that the rank of $\left(s_{i j}\right)$ is less than $n+1$ so that $\left|\left(s_{i j}\right)\right|=0$ and $\left(s_{i j}\right)$ is semi-positive definite. Further we note that both $\left(s_{i j}\right)$ and $\left(w_{i j}\right)$ are symmetric matrices and since $B_{1}, \cdots, B_{n+1}$ do not lie on a $(n-1)$-dimensional subspace, we must have $\left|\left(w_{i j}\right)\right| \neq 0$, in fact, $\left|\left(w_{i j}\right)\right|>0$ since $\left(w_{i j}\right)$ is positive definite. Our problem of maximizing $C\left(S_{n}\right)$ with respect to the $l_{i j}, i \neq j$, for given values of $d_{i}, d_{i}>0$, may be re-stated as follows.

We must maximize $\left|\left(w_{i j}\right)\right|$ over the class of symmetric matrices $\left(s_{i j}\right)$ or $\left(w_{i j}\right)$ with respect to $s_{i j}, i, j=1, \cdots, n+1$, subject to the conditions: $\left|\left(s_{i j}\right)\right|=0$ and $s_{i i}=d_{i}^{2}$ for $i=1, \cdots, n+1$. Further $\left(s_{i j}\right)$ should be semipositive definite and $\left|w_{i j}\right| \neq 0$.

Let $\theta$ and $\mu_{1}, \cdots, \mu_{n+1}$ be Lagrange multipliers. We seek the extreme values of the function $L$ with respect to $s_{i j}, i, j=1, \cdots$, $n+1$, where

$$
L=\left|w_{i j}\right|-\theta\left|s_{i j}\right|+\sum_{i=1}^{n+1} \mu_{i}\left(s_{i i}-d_{i}^{2}\right) .
$$

Hence $s_{i j}$ must satisfy

$$
\begin{aligned}
& \frac{1}{2} \frac{\partial L}{\partial s_{i j}}=\left|W_{i j}\right|-\theta\left|S_{i j}\right|=0 \text { for } i \neq j, i, j,=1, \cdots, n+1 \\
& \text { and } \frac{\partial L}{\partial s_{i i}}=\left|W_{i i}\right|-\theta\left|S_{i i}\right|+\mu_{i}=0 \text { for } i=1, \cdots, n+1 ;
\end{aligned}
$$

where $\left|W_{k l}\right|$ and $\left|S_{k l}\right|$ denote co-factors of $w_{k l}$ and $s_{k l}$ in $\left|\left(w_{i j}\right)\right|$ and $\left|\left(s_{i j}\right)\right|$ respectively.

This implies that

$$
\sum_{j=1}^{n+1} w_{k j} \cdot \frac{1}{2} \frac{\partial L}{\partial s_{i j}}+w_{k i} \frac{\partial L}{\partial s_{i i}}=0
$$

so that

$$
\sum_{j=1}^{n+1} w_{k j}\left|W_{i j}\right|-\theta \sum_{j=1}^{n+1} w_{k j}\left|S_{i j}\right|+\mu_{i} w_{k i}=0 .
$$

Let $k \neq i$; then using (2.2), $w_{k j}=1+s_{k j}$ and by the well-known property that expansions in terms of alien co-factors vanish identically (Aitken, p. 51) we finally obtain

$$
-\theta \sum_{j=1}^{n+1}\left|S_{i j}\right|+\mu_{i} w_{k i}=0
$$

so that $s_{k i}=w_{k i}-1=\theta / \mu_{i} \sum_{j=1}^{n+1}\left|S_{i j}\right|-1$, for all $k \neq i$. Since the above expression for $s_{k i}$ is constant for values of $k=1, \cdots, n+1$, $k \neq i$, we conclude that the elements of the $i$ th column of $\left(s_{i j}\right)$, except 
$s_{i i}=d_{i}^{2}$, must be equal. Since $s_{i j}$ is a symmetric matrix, the above property extends to the rows of $\left(s_{i j}\right)$ and it is easily seen that the extreme values of $L$ correspond to values $s_{i j}^{*}$ of $s_{i j}$ where

$$
s_{i j}^{*}=u \text { for } i \neq j, i, j=1, \cdots, n+1
$$

while

$$
s_{i i}^{*}=d_{i}^{2}, i=1, \cdots, n+1 .
$$

Now $u$ can be determined from the relation $\left|s_{i j}\right|=0$ so that we must have

$$
\left|\begin{array}{lllll}
d_{1}^{2} & u & \cdot & \cdot & u \\
u & d_{2}^{2} & \cdot & \cdot & u \\
\cdot & \cdot & \cdot & \cdot & \cdot \\
u & u & \cdot & \cdot & d_{n+1}^{2}
\end{array}\right|=0 .
$$

Let us define the determinant

$$
D_{k}\left(x ; a_{1}, \cdots, a_{k}\right)=\left|\begin{array}{ccccc}
a_{1} & x & \cdot & \cdot & x \\
x & a_{2} & \cdot & \cdot & x \\
\cdot & \cdot & \cdot & \cdot & \cdot \\
x & x & \cdot & \cdot & a_{k}
\end{array}\right| .
$$

From the relation due to Grabeiri (1874) (see Muir, vol. 3, 4, p. 110), or by subtracting the first row of the above determinant from the remaining rows and by the use of Cauchy expansion in terms of the first row and first column, we have

$$
D_{k}\left(x ; a_{1}, \cdots, a_{k}\right)=\left(1+x \sum_{i=1}^{k}\left(a_{i}-x\right)^{-1}\right) \prod_{i=1}^{k}\left(a_{i}-x\right) .
$$

Hence from (2.6) $u$ must satisfy the equation

$$
\left(1+u \sum_{i=1}^{n+1}\left(d_{i}^{2}-u\right)^{-1}\right) \prod_{i=1}^{n+1}\left(d_{i}^{2}-u\right)=0 .
$$

From (2.2) and (2.5) the extreme value of $\left(n ! C\left(S_{n}\right)\right)^{2}$ for any $u$ satisfying $(2.9)$ is equal to

$$
\begin{aligned}
& D_{n+1}\left(1+u ; 1+d_{1}^{2}, \cdots, 1+d_{n+1}^{2}\right) \\
= & \left(1+(1+u) \sum_{i=1}^{n+1}\left(d_{i}^{2}-u\right)^{-1}\right)\left(\prod_{i=1}^{n+1}\left(d_{i}^{2}-u\right)\right) \\
= & \left.\left(\sum_{i=1}^{n+1}\left(d_{i}^{2}-u\right)^{-1}\right)\left(\prod_{i=1}^{n+1} d_{i}^{2}-u\right)\right)
\end{aligned}
$$

by the use of (2.9).

Since $u=0$ does not satisfy (2.6), we immediately obtain from 
(2.9) that the expression (2.10) is equal to

$$
-u^{-1} \prod_{i=1}^{n+1}\left(d_{i}^{2}-u\right)
$$

which is the extreme value of $\left(n ! C\left(s_{n}\right)\right)^{2}$ in terms of $u$. In order that the content is nonzero we must have $u \neq d_{i}^{2}$ for $i=1, \cdots, n+1$. This statement along with (2.9) implies that $u$ must satisfy the equation

$$
1+u \sum_{i=1}^{n+1}\left(d_{i}^{2}-u\right)^{-1}=0 .
$$

The roots for $u$, temporarily assuming that $d_{1}, \cdots, d_{n+1}$ are distinct, can be located by Decartes rule of signs by checking the signs of the left-handside of (2.12) for values of $u$, equal to $-\infty, 0,+\infty$ and in the neighborhood of $d_{i}^{2}, i=1, \cdots, n+1$. Relabelling $d_{i}$ such that $d_{1}<d_{2}<\cdots<d_{n+1}$, it is easily verified that all the roots for $u$ are real, say $u_{1}, \cdots, u_{n+1}$ and may be labelled in such a manner that

$$
u_{1}<0<d_{1}^{2}<u_{2}<d_{2}^{2}<\cdots<u_{n+1}<d_{n+1}^{2} .
$$

Consider the characteristic roots of $\left(s_{i j}^{*}\right)$ given by $\left|s_{i j}^{*}-\lambda I\right|=0$. By (2.5) and (2.7) $\lambda$ must satisfy $D_{n+1}\left(u ; d_{1}^{2}-\lambda, \cdots, d_{n+1}^{2}-\lambda\right)=0$. Hence from $(2.9)$

$$
\left(1+u \sum_{i=1}^{n+1}\left(d_{i}^{2}-\lambda-u\right)^{-1}\right) \prod_{i=1}^{n+1}\left(d_{i}^{2}-\lambda-u\right)=0 .
$$

By similar method as used to obtain (2.13) we find that the roots for $\lambda$ may be so labelled that $\lambda_{1}=0$ and

$$
d_{i}^{2}<\lambda_{i+1}+u<d_{i+1}^{2} \quad i=1, \cdots, n .
$$

In order that all the roots for $\lambda$ are nonnegative it is easily seen that the relation

$$
d_{2}^{2}-u>\lambda_{2} \geqq 0
$$

must be satisfied so that we must have $u<d_{2}^{2}$. From (2.13) we find that the only admissible roots for $u$ are $u_{1}$ and $u_{2}$.

To establish (1.4) it only remains to show that $u_{1}$ corresponds to the case when $A$ is inside the extremal simplex whereas $u_{2}$ corresponds to the case when $A$ is outside the extremal simplex.

Consider the equation of $\pi_{i}$, passing through all the vertices of $S_{n}$ except $B_{i}$ having the coordinates $\left(x_{i, 1}, \cdots, x_{i, n}\right)$, given by

$$
L_{i}\left(x_{1}, \cdots, x_{n}\right)=0 \text {, }
$$

where 


$$
L_{i}\left(x_{1}, \cdots, x_{n}\right)=\left|\begin{array}{cccc}
x_{1,1} & \cdots & x_{1, n} & 1 \\
\vdots & & \vdots & \vdots \\
x_{i-1,1} & \cdots & x_{i-1, n} & 1 \\
x_{1} & \cdots & x_{n} & 1 \\
x_{i+1,1} & \cdots & x_{1+1, n} & 1 \\
\vdots & & \vdots & \vdots \\
x_{n+1,1} & \cdots & x_{n+1, n} & 1
\end{array}\right|
$$

Now $A$ and $B_{i}$ lie on the same side of $\pi_{i}$ if and only if $L_{i}\left(x_{i, 1}, \cdots, x_{i, n}\right)$. $L_{i}(0, \cdots, 0)>0$ while $A$ and $B_{i}$ lie on opposite sides of $\pi_{i}$ if and only if $L_{i}\left(x_{i, 1}, \cdots, x_{i, n}\right) . \quad L_{i}(0, \cdots, 0)<0$.

Now by direct multiplication of the determinant $L_{i}\left(x_{i, 1}, \cdots, x_{i, n}\right)$ with the transpose of the determinant $L_{i}(0,0, \cdots, 0)$ we obtain

$$
\begin{aligned}
& L_{i}\left(x_{i, 1}, \cdots, x_{i, n}\right) \cdot L_{i}(0,0, \cdots, 0)
\end{aligned}
$$

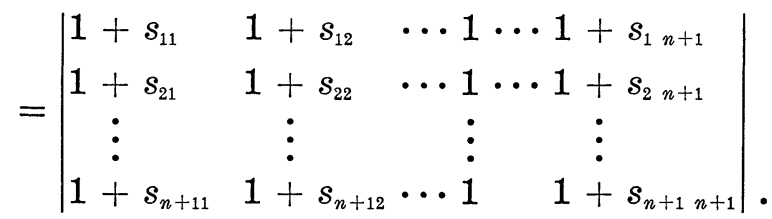

We now assume that $S_{n}$ is an extremal simplex so that from (2.5) $s_{\nu \nu}=d_{\nu}^{2}, \nu=1, \cdots, n+1$ and $s_{\nu k}=u, \nu \neq k, \nu, k=1, \cdots, n+1$. Then in the last determinant each entry in the $i$-th column is 1 , the $j$ th diagonal entry is $d_{j}^{2}+1$ for $j \neq i, j=1, \cdots, n+1$ while the remaining entries are $1+u$. Subtracting $(1+u)$ times the $i$-th column from the remaining columns we immediately obtain

$$
\begin{aligned}
L_{i}\left(x_{i, 1}, \cdots, x_{i, n}\right) \cdot L_{i}(0, \cdots, 0) & =\left(d_{i}^{2}-u\right)^{-1} \prod_{j=1}^{n+1}\left(d_{j}^{2}-u\right) \\
& =\frac{-u^{-1} \prod_{1}^{n+1}\left(d_{j}^{2}-u\right)}{\left(-u^{-1}\left(d_{i}^{2}-u\right)\right)}
\end{aligned}
$$

Since from (2.11) the numerator of the last expression is positive, we find that $A$ and $B_{i}$ lie on the same side of $\pi_{i}$ if and only if

$$
-u^{-1}\left(d_{i}^{2}-u\right)>0
$$

while they lie on opposite sides of $\pi_{i}$ if and only if $-u^{-1}\left(d_{i}^{2}-u\right)<0$.

Since $-u_{2}^{-1}\left(d_{2}^{2}-u_{2}\right)<0$ and $-u_{1}^{-1}\left(d_{1}^{2}-u_{1}\right)>0$, it is readily checked that we have proved (1.2), (1.3) and (1.4) in the case when $d_{1}, \cdots, d_{n+1}$ are distinct.

Necessary modifications are easily made when some or all of the $d_{i}$ are not distinct.

Finally we remark that the simplex corresponding to $u_{1}$ has larger 
content than that for $u_{2}$. This is because

$$
d_{i}^{2}-u_{1}>d_{i}^{2}-u_{2}>0 \text { for } i=2, \cdots, n-1
$$

and

$$
-u_{1}^{-1}\left(d_{1}^{2}-u_{1}\right)=1-\frac{d_{1}^{2}}{u_{1}}>1-d_{1}^{2} / u_{2}=-u_{2}^{-1}\left(d_{2}^{2}-u_{2}\right),
$$

so that

$$
-u_{1}^{-1} \prod_{1}^{n+1}\left(d_{j}^{2}-u_{1}\right)>-u_{2}^{-1} \prod_{1}^{n+1}\left(d_{j}^{2}-u_{2}\right) .
$$

We also note that when $d_{1}=d_{2}=\cdots=d_{n+1}$ (1.4) has a unique negative root for $u$ and the point $A$ corresponding to this value of $u$ must lie inside the extremal simplex.

3. Simplex determined by distances of faces. We recall that the $(n-1)$-dimensional hyperplane $\pi_{i}$ passes through all the vertices of $S_{n}$ except $B_{i}$. The distance of $\pi_{i}$ from $A$ is $p_{i}$. The point $B_{i}$ does not lie on $\pi_{i}$ but does lie on all the remaining $n$ hyperplanes

$$
\pi_{j}, j \neq i, j=1, \cdots, n+1 \text {. }
$$

Let $\pi_{i}$ be given by (in normal form)

$$
\pi_{i}: e_{i, 1} x_{1}+e_{i, 2} x_{2}+\cdots+e_{i, n} x_{n}=e_{i, n+1}
$$

where for notational convenience we have written

$$
p_{i}=e_{i, n+1},
$$

and $e_{i, 1}, \cdots, e_{i}, n$ are the direction cosines of the normal to $\pi_{i}$, so that we have

$$
\sum_{j=1}^{k} e_{i, j} e_{k, j}=m_{i k} ; i, k=1,2, \cdots, n+1 ; m_{i i}=1 .
$$

The notations used in this section will be listed first and some relations needed later will be established in order to avoid future digression.

We define the $(n+1) \times(n+1)$ matrix $E$ in double suffix notation as

$$
E=\left(e_{i, j}\right)
$$

and $E_{i, j}$ will denote the co-factor of $e_{i, j}$ in $E$. We also define the $(n+1) \times(n+1)$ matrix $M$ as

$$
M=\left(m_{i j}\right)
$$


and $M_{i j}$ as co-factor of $m_{i j}$ in $M$.

Let $\sigma_{i}$ denote the signature of $\left|E_{i, n+1}\right|$ so that

$$
\sigma_{i}=\left\{\begin{array}{c}
1 \text { if }\left|E_{i, n+1}\right|>0 \\
-1 \text { if }\left|E_{i, n+1}\right|<0
\end{array} \text { for } i=1, \cdots, n+1 .\right.
$$

We remark here that $E_{i, n+1}$ is nonsingular. This is because

$$
\pi_{1}, \cdots, \pi_{i-1}, \pi_{i+1}, \cdots, \pi_{n+1}
$$

have one and only one point in common, namely $\left(x_{i, 1}, \cdots, x_{i, n}\right)$. Since $\pi_{i}$ does not pass through the above common point, it is easily seen that the matrix $E$ is also nonsingular, so that

$$
|E| \neq 0 \text { and }\left|E_{i, n+1}\right| \neq 0, i=1, \cdots, n+1 .
$$

Furthermore it is easily seen that

$$
\begin{aligned}
& \left|E_{i, n+1}\right|=\sigma_{i}\left|E_{i, n+1} E_{i, n+1}^{\prime}\right|^{1 / 2}=\sigma_{i}\left|M_{i i}\right|^{1 / 2} \\
& \qquad \text { for } i=1, \cdots, n+1
\end{aligned}
$$

where the radical above as well as all radicals appearing in this paper will be always taken as positive. Hence from (3.2) and (3.4) we have

$$
|E|=\sum_{i=1}^{n+1} p_{i}\left|E_{i, n+1}\right|=\sum_{i=1}^{n+1} \sigma_{i} p_{i}\left|M_{i i}\right|^{1 / 2}=\rho \text { (say) . }
$$

$D$ will denote the diagonal matrix

$$
D=\text { Diag. }\left(p_{1}, \cdots, p_{n+1}\right)
$$

and let

$$
R=\left(r_{i j}\right)=D^{-1} M D^{-1}
$$

so that $r_{i i}=p_{i}^{-2}$ for $i=1, \cdots, n+1$. Since

$$
M=\left[\begin{array}{ccc}
e_{1,1} & \cdots & e_{1, n} \\
\vdots & & \vdots \\
e_{n+1,1} & \cdots & e_{n+1, n}
\end{array}\right]\left[\begin{array}{ccc}
e_{1,1} & \cdots & e_{1, n} \\
\vdots & & \vdots \\
e_{n+1,1} & \cdots & e_{n+1, n}
\end{array}\right]^{\prime}
$$

we also remark that $M$ and consequently $R$ are symmetric positive semi-definite matrices, so that $|M|=0$ and $|R|=0$.

Finally, it follows that

$$
\left|M_{i i}\right|=\left|R_{i i}\right|\left(\prod_{j=1}^{n+1} p_{j}^{2}\right) / p_{i} .
$$

To obtain the content $C\left(S_{n}\right)$, we will use the formula (2.1). Since $\left(x_{i, 1}, \cdots x_{i, n}\right)$ lies on $\pi_{j} ; j \neq i, j=1, \cdots, n+1$, we may directly solve for $x_{i, j}$ from the following $n$ linear equations: 


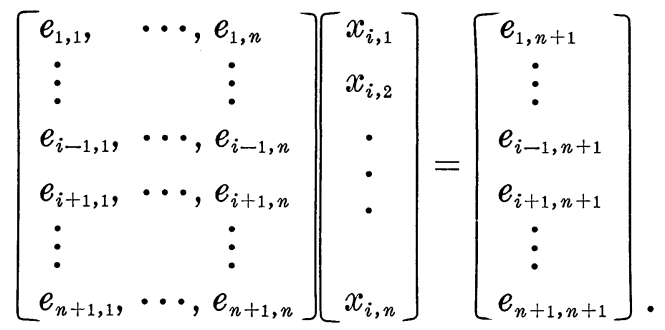

A simple calculation shows that (see (3.4))

$$
x_{i, j}=(-1)^{n-j}(-1)^{i+j}\left|E_{i, j}\right| /\left((-1)^{n+1+i}\left|E_{i, n+1}\right|\right) .
$$

Hence we obtain

$$
x_{i, j}=-\left|E_{i, j}\right| /\left|E_{i, n+1}\right| ; i, j=1, \cdots, n+1 .
$$

Substituting these values in $|V|$ of (2.1) and factoring out -1 from each of the first $n$ columns of $V$ and also factoring out $\left|E_{i, n+1}\right|^{-1}$ from the $i$ th row of $V$ for $i=1, \cdots, n+1$, we readily obtain

$$
\begin{aligned}
n ! C\left(S_{n}\right) & =(-1)^{n}|\operatorname{Adj} E| / \prod_{i=1}^{n+1}\left|E_{i, n+1}\right| \\
& =(-1)^{n}|E|^{n} / \prod_{i=1}^{n+1}\left|E_{i, n+1}\right|
\end{aligned}
$$

where $|\operatorname{Adj} E|$ is the adjoint determinant of $|E|$. In order to avoid the ambiguity of sign in $C\left(S_{n}\right)$ we consider $\left(n ! C\left(S_{n}\right)\right)^{2}$ instead and from (3.9) and (3.12) we obtain

$$
\begin{aligned}
\left(n ! C\left(S_{n}\right)\right)^{2} & =|E|^{2 n} / \prod_{i=1}^{n+1}\left|E_{i, n+1}\right|^{2} \\
& =\left(\sum_{i=1}^{n+1} \sigma_{i} p_{i}\left|M_{i i}\right|^{1 / 2}\right)^{2 n} / \prod_{i=1}^{n+1}\left|M_{i i}\right| \\
& =\left(\sum_{i=1}^{n+1} \sigma_{i}\left|R_{i i}\right|^{1 / 2}\right)^{2 n} / \prod_{i=1}^{n+1}\left|R_{i i}\right| .
\end{aligned}
$$

Our problem of minimization is equivalent to minimizing

$$
\ln \left[\left(\sum_{i=1}^{n+1} \sigma_{i}\left|R_{i i}\right|^{1 / 2}\right)^{2} / \prod_{i=1}^{n+1}\left|R_{i i}\right|^{1 / n}\right]
$$

with respect to $r_{i j}, i, j=1, \cdots, n+1$, subject to the restriction that $r_{i i}=p_{i}^{-2}, i=1, \cdots, n+1$ and $|R|=0$ over the class of symmetric matrices $R$.

Let $\lambda, \mu_{1}, \cdots, \mu_{n+1}$ be Lagrange multipliers and we seek the extreme value of

$$
L=\ln \left(\sum_{i=1}^{n+1} \sigma_{i}\left|R_{i i}\right|^{1 / 2}\right)^{2}-\frac{1}{n} \sum_{i=1}^{n+1} \ln \left|R_{i i}\right|-\lambda|R|+\sum_{i=1}^{n+1} \mu_{i}\left(r_{i i}-p_{i}^{-2}\right) .
$$


$r_{i j}$ must satisfy:

$$
\begin{array}{r}
\frac{\partial L}{\partial r_{i j}}=\rho^{-1} \sum_{\nu=1}^{n+1} \frac{\partial\left|R_{\nu \nu}\right|}{\partial r_{i j}} \frac{\sigma_{\nu}}{\left|R_{\nu \nu}\right|^{1 / 2}}-\frac{1}{n} \sum_{\nu=1}^{n+1} \frac{1}{R_{\nu \nu}} \frac{\partial\left|R_{\nu \nu}\right|}{\partial r_{i j}}-\lambda \frac{\partial|R|}{\partial r_{i j}}=0, \\
i \neq j, i, j=1, \cdots, n+1
\end{array}
$$

and

$$
\frac{\partial L}{\partial r_{i i}}=\rho^{-1} \sum_{\nu=1}^{n+1} \frac{\sigma_{\nu}}{\left|R_{\nu \nu}\right|^{1 / 2}} \frac{\partial\left|R_{\nu \nu}\right|}{\partial r_{i i}}-\frac{1}{n} \sum_{\nu=1}^{n+1} \frac{1}{R_{\nu \nu}} \frac{\partial\left|R_{\nu \nu}\right|}{\partial r_{i i}}-\lambda \frac{\partial|R|}{\partial r_{i i}}+\mu_{i}=0
$$

where $\rho$ is as defined in (3.9).

These equations reduce to

$$
\begin{array}{r}
\frac{1}{2} \frac{\partial L}{\partial r_{i j}}=\sum_{\substack{\nu=1 \\
\nu \neq i, j}}^{n+1}\left(\rho^{-1} \sigma_{\nu}\left|R_{\nu \nu}\right|^{-1 / 2}-n^{-1}\left|R_{\nu \nu}\right|^{-1}\right)\left|R_{\nu \nu \mid i j}\right|-\lambda\left|R_{i j}\right|=0 \\
\qquad \text { for } i \neq j ; i, j=1, \cdots, n+1
\end{array}
$$

and

$$
\frac{\partial L}{\partial r_{i i}}=\sum_{\substack{\nu=1 \\ \nu \neq i}}^{n+1}\left(\rho^{-1} \sigma_{\nu}\left|R_{\nu \nu}\right|^{-1 / 2}-n^{-1}\left|R_{\nu \nu}\right|^{-1}\right)\left|R_{\nu \nu \mid i i}\right|-\lambda\left|R_{i i}\right|+\mu=0
$$

where $\left|R_{\nu \nu \mid i j}\right|$ is the co-factor of $r_{i j}$ in $\left|R_{\nu \nu}\right|$.

Hence the minimizing values of $r_{i j}, r_{i j}^{*}$, say, must satisfy the equations in $r_{i j}$ :

$$
r_{i i}=p_{i}^{-2}
$$

and

$$
\sum_{\substack{j=1 \\ j \neq i}}^{n+1} r_{i j} \frac{1}{2} \frac{\partial L}{\partial r_{i j}}+r_{i i} \frac{\partial L}{\partial r_{i i}}=0
$$

and

$$
\sum_{\substack{j=1 \\ j \neq i}}^{n+1} r_{k j} \frac{1}{2} \frac{\partial L}{\partial r_{i j}}+r_{k j} \frac{\partial L}{\partial r_{i i}}=0
$$

After obvious simplification (3.14) yields

$$
\sum_{\substack{\nu=1 \\ \nu \neq i}}^{n+1}\left(\rho^{-1} \sigma_{\nu}\left|R_{\nu \nu}\right|^{-1 / 2}-n^{-1}\left|R_{\nu \nu}\right|^{-1}\right)\left|R_{\nu \nu}\right|+\mu_{i} p_{i}^{-2}=0
$$

or

$$
\mu_{i}=p_{i}^{2} \rho^{-1} \sigma_{i} R_{i i}
$$

From (3.15) we obtain for $k \neq i$, 


$$
\sum_{j=1}^{n+1} \sum_{\substack{\nu=1 \\ \nu \neq i, j}}^{n+1}\left(\sigma_{\nu}\left|R_{\nu \nu}\right|^{-1 / 2} \rho^{-1}-n^{-1}\left|R_{\nu \nu}\right|^{-1}\right) r_{k j}\left|R_{\nu \nu \mid i j}\right|+\mu_{i} r_{k i}=0
$$

After some calculations we obtain

$$
r_{k i}=\mu_{i}^{-1}\left(\sigma_{k}\left|R_{k k}\right|^{-1 / 2} \rho^{-1}-n^{-1}\left|R_{k k}\right|^{-1}\right)\left|R_{i k}\right| .
$$

It is easily seen from (3.11) that $\left|R_{i k}\right|=p_{i} p_{k}\left|M_{i k}\right|$ and

$$
M_{i k}=\left|E_{i, n+1}\right|\left|E_{k, n+1}\right|
$$

and hence from (3.8),

$$
\left|R_{i k}\right|=\sigma_{i k}\left|R_{i i}\right|^{1 / 2}\left|R_{k k}\right|^{1 / 2}
$$

so that substituting for $\mu_{i}$ from (3.16) in (3.18) we obtain

$$
p_{i}^{2} r_{k i}=1-n^{-1} \rho \sigma_{k}\left|R_{k k}\right|^{-1 / 2} .
$$

In obtaining (3.18) from (3.17), we illustrate the case for $i=1$, $n+1=4$ and $k=2$, for the expression, for example:

$$
\begin{aligned}
& \sum_{j=1}^{4} \sum_{\substack{\nu=1 \\
\nu \neq 1, j}}^{4} \sigma_{\nu}\left|R_{\nu \nu}\right|^{-1 / 2} r_{k j}\left|R_{\nu \nu \mid i j}\right| \\
& =r_{21}\left(\sigma_{2}\left|R_{22 \mid 11}\right|\left|R_{22}\right|^{-1 / 2}+\sigma_{3}\left|R_{33 \mid 11}\right|\left|R_{33}\right|^{-1 / 2}+\sigma_{4}\left|R_{44 \mid 11}\right|\left|R_{44}\right|^{-1 / 2}\right) \\
& \quad+r_{22}\left(\sigma_{3}\left|R_{33 \mid 12}\right|\left|R_{33}\right|^{-1 / 2}+\sigma_{4}\left|R_{44 \mid 12}\right|\left|R_{44}\right|^{-1 / 2}\right) \\
& \quad+r_{23}\left(\sigma_{2}\left|R_{22 \mid 13}\right|\left|R_{22}\right|^{-1 / 2}+\sigma_{4}\left|R_{44 \mid 13}\right|\left|R_{44}\right|^{-1 / 2}\right) \\
& \quad+r_{24}\left(\sigma_{2}\left|R_{22 \mid 14}\right|\left|R_{22}\right|^{-1 / 2}+\sigma_{3}\left|R_{33 \mid 14}\right|\left|R_{33}\right|^{-1 / 2}\right) \\
& = \\
& =\sigma_{2}\left|R_{21}\right|\left|R_{22}\right|^{-1 / 2} .
\end{aligned}
$$

The last expression is obtained from the coefficients of $\left|R_{22}\right|^{-1 / 2}$; the coefficients of $\left|R_{33}\right|^{-1 / 2}$ or $\left|R_{11}\right|^{-1 / 2}$ are easily seen to vanish identically, since they represent expansion by alien co-factors.

In the summation appearing in (3.17) only the term with $\nu=k$ survives;

$$
\sum_{\substack{j=1 \\ j \neq k}}^{n+1} r_{k j}\left|R_{k k \mid i j}\right|
$$

is the expansion of the determinant obtained by replacing the elements of the $i$-th row of $|R|$ by those of the $k$-th row of $|R|$ with the $k$-th row and $k$-th column deleted. Transferring the elements $r_{k i}$ appearing in the $i$-th row to the $k$-th row, there results the minor of $r_{k i}$ in $|R|$. Hence multiplying by $(-1)^{i-k}$ and $(-1)^{i+k}$ we obtain $\left|R_{k i}\right|$. It is thus seen that

$$
\sum_{\substack{j=1 \\ j \neq k}}^{n+1} r_{k j}\left|R_{k k i i j}\right|=\left|R_{k i}\right|=\left|R_{i k}\right|
$$


From (3.19) it is easily checked that we have

$$
p_{i}^{2} p_{k}^{2} r_{i k}=p_{j}^{2} p_{k}^{2} r_{j k},
$$

for all $i, j=1, \cdots, n+1$, with $i \neq k, j \neq k$.

Since the matrix

$$
\left(p_{i}^{2} r_{i j} p_{j}^{2}\right)=D^{2} R D^{2}=D^{2} D^{-1} M D^{-1} D^{2}=D M D=\left(p_{i} m_{i j} p_{j}\right)
$$

is symmetric, and (3.20) implies that nondiagonal elements of each row or column of this matrix are equal we conclude, (in a manner analogous to (2.5)) that $r_{i i}^{*}=p_{i}^{-2}, i=1, \cdots, n+1$ and

$$
p_{i}^{2} r_{i j}^{*} p_{j}^{2}=p_{i} p_{j} m_{i j}^{*}=v \text {, }
$$

say, for $i \neq j ; i, j=1, \cdots, n+1$ so that

$$
\left\{\begin{array}{l}
m_{i i}^{*}=1 \quad \text { for } i=1, \cdots, n+1 \\
m_{i j}^{*}=\frac{v}{p_{i} p_{j}} \text { for } i \neq j ; i, j=1, \cdots, n+1 .
\end{array}\right.
$$

We obtain values of $v$ by equating $\left|r_{i j}^{*}\right|=0$ or equivalently by setting $|D M D|=\left|\left(p_{i} p_{j} m_{i j}^{*}\right)\right|=0$, where $p_{i} p_{j} m_{i j}^{*}=v, i \neq j$ and $p_{i}^{2} m_{i i}^{*}=p_{i}^{2}$, and it is seen from (2.7) that $v$ must satisfy

$$
D_{n+1}\left(v ; p_{1}^{2}, \cdots, p_{n+1}^{2}\right)=0 \text {. }
$$

and hence

$$
\left(1+v \sum_{i=1}^{n+1}\left(p_{i}^{2}-v\right)^{-1}\right) \prod_{i=1}^{n+1}\left(p_{i}^{2}-v\right)=0 .
$$

We also note from (3.13), (3.8), (3.9) and (3.12) that

$$
\begin{aligned}
\left(n ! C\left(S_{n}\right)\right)^{2} & =\rho^{2 n} \cdot \prod_{i=1}^{n+1}\left(\left|R_{i i}\right|^{-1} \cdot p_{i}^{2}\right) \\
& =p_{1}^{2}\left|R_{11}\right|^{-1} \cdot \prod_{i=2}^{n+1}\left(\rho\left|R_{i i}\right|^{-1 / 2}\right)^{2} .
\end{aligned}
$$

But from (3.19) we have

$$
\rho \sigma_{k}\left|R_{k k}\right|^{-1 / 2}=n\left(1-p_{i}^{2} r_{k i}^{*}\right)
$$

so that $\rho \sigma_{k}\left|R_{i i}\right|^{-1 / 2}=n\left(p_{i}^{2}-v\right) / p_{i}$, from (3.21). Also from (3.21), since $r_{i j}^{*}=v /\left(p_{i}^{2} p_{j}^{2}\right)$ and $r_{i i}^{*}=p_{i}^{-2}$ it is easily seen that

$$
\begin{aligned}
\left|R_{11}\right| \prod_{i=2}^{n+1} p_{i}^{2} & =D_{n}\left(v ; p_{2}^{2}, \cdots, p_{n+1}^{2}\right) \\
& =\left(1+v \sum_{i=2}^{n+1}\left(p_{i}^{2}-v\right)^{-1}\right) \prod_{i=2}^{n+1}\left(p_{i}^{2}-v\right) \\
& =\left(p_{1}^{2}-v\right)^{-1}\left(-v\left(p_{1}^{2}-v\right)^{-1}+\right.
\end{aligned}
$$




$$
\begin{aligned}
& \left.+1+v \sum_{i=1}^{n+1}\left(p_{1}^{2}-v\right)^{-1}\right) \prod_{i=1}^{n+1}\left(p_{i}^{2}-v\right) \\
& =-v\left(p_{1}^{2}-v\right)^{-2} \prod_{i=1}^{n+1}\left(p_{i}^{2}-v\right) \text { from } \quad(3.22)
\end{aligned}
$$

Substituting in (3.23) we readily find that

$$
\left(n ! C\left(S_{n}\right)\right)^{2}=v^{-1} n^{2 n} \prod_{i=1}^{n+1}\left(p_{i}^{2}-v\right) .
$$

Thus (1.6) is proved.

In order that $S_{n}$ is nondegenerate $v \neq p_{i}^{2}, i=1, \cdots, n+1$. Hence from (3.22) $v$ must satisfy

$$
1+v \sum_{i=1}^{n+1}\left(p_{i}^{2}-v\right)^{-1}=0 .
$$

Thus we have exactly the same equation as (2.12) with $d_{i}$ replaced by $p_{i}$ and $u$ replaced by $v$. By exactly the same argument that follows (2.12) we conclude that, when $p_{1}, \cdots, p_{n+1}$ are distinct, if the roots of (3.25) are so labelled that the unique negative root of (3.25) is $v_{1}$ and the smallest positive root for $v$ is $v_{2}$ and if the $p_{i}$ are labelled so that $p_{1}$ is the smallest and $p_{2}$ the second smallest $p_{i}, i=1, \cdots, n+1$, we have the two eligible roots of (3.26) as $v_{1}$ and $v_{2}$ satisfying

$$
v_{1}<0<p_{1}^{2}<v_{2}<p_{2}^{2} \text {. }
$$

It remains to prove that $v_{1}$ corresponds to the case when $A$ is inside $S_{n}$ while $v_{2}$ corresponds to the case when $A$ is outside $S_{n}$.

We will prove that, for the extremal simplexes obtained above, the vertex $B_{i}$ and the fixed point $A$ lie on the same side of $\pi_{i}$ if

$$
p_{i}^{2}-v>0
$$

while $A$ and $B_{i}$ lie on opposite sides if $p_{i}^{2}-v<0$.

Let

$$
L_{i}\left(x_{1}, \cdots, x_{n}\right)=e_{i, 1} x_{1}+\cdots+e_{i, n} x_{n}-e_{i, n+1} .
$$

Then $L_{i}(0, \cdots, 0)=-e_{i, n+1}=-p_{i}$, and

$$
\begin{array}{rlrl}
L_{i}\left(x_{i 1}, \cdots, x_{i n}\right) & & \\
& =-\sum_{j=1}^{n+1} e_{i, j}\left|E_{i, j}\right| /\left|E_{i, n+1}\right| & & \text { (by virtue of (3.5)) } \\
& =-|E| /\left|E_{i, n+1}\right| & & \\
& =-p_{i} \rho / \sigma_{i}\left|R_{i i}\right|^{1 / 2} & & \text { from (3.8) and (3.1 ) } \\
& =-n p_{i}\left(1-p_{k}^{2} r_{k i}^{*}\right) & & \text { (from (3.19)) } \\
& =-n p_{i}\left(1-v / p_{i}^{2}\right) & & \text { (from (3.21)) }
\end{array}
$$


Hence $L_{i}(0, \cdots, 0) \cdot L_{i}\left(x_{i, 1}, \cdots, x_{i, n}\right)=n\left(p_{i}^{2}-v\right)$. Now the equation of $\pi_{i}$ is $L_{i}\left(x_{1}, \cdots, x_{n}\right)=0$. Hence $p_{i}^{2}-v>0$ implies that $A$ and $B_{i}$ lie on the same side of $\pi_{i}$ while $p_{i}^{2}-v<0$ implies that $A$ and $B_{i}$ lie on opposite sides of $\pi_{i}$. Since $p_{i}^{2}-v_{1}$ is positive for $i=1, \cdots, n+1$ we conclude from (3.26) that corresponding to $v_{1}, A$ is inside $S_{n}$. Also from (3.26) we find $p_{1}^{2}-v_{2}$ is negative so that corresponding to $v_{2}$ the point $A$ lies outside $S_{n}$. Hence it is readily checked that we have proved (1.5), (1.6), (1.7) and (1.8).

Finally, using an argument analogous to that used to obtain (2.15) we find that

$$
-v_{1}^{-1} \prod_{i=1}^{n+1}\left(p_{i}^{1}-v_{1}\right)>-v_{2}^{-1} \prod_{i=1}^{n+1}\left(p_{i}^{2}-v_{2}\right)
$$

so that from (3.24) we conclude that the content of $S_{n}$ corresponding to $v_{1}$ is greater than the content of $S_{n}$ corresponding to $v_{2}$.

Obvious modifications in the foregoing proofs are easily made. when some or all the $p_{1}, \cdots, p_{n+1}$ are equal.

When $p_{1}=p_{2}=\cdots=p_{n+1}$, (3.25) has a unique negative solution for $v$ and in this case $A$ must lie inside the extremal simplex.

The author expresses his thanks to Professor H. S. M. Coxeter for his valuable association which led to this problem and for his keen interest in this work.

\section{REFERENCES}

1. A. C. Aitken, Determinants and matrices, Oliver and Boyd, Edinburgh, 1956.

2. T. Muir, The theory of determinants in the historical order of development, Volume 3, 4, Dover Publications Inc., 1920.

3. D. M. Y. Sommerville, An introduction to the geometry of $N$ dimensions, Methuen, London, 1929.

4. D. Slepian, The content of some extreme simplexes, Pacific J. Math., (to appear).

Received July 23, 1969. This work was done at the University of Toronto, Department of Industrial Engineering, and the author was on leave of absence from the University of Western Ontario, Department of Mathematics.

UNiVERSity OF WESTERN ONTARIO, AND

UNIVERSITY OF TORONTO 


\section{PACIFIC JOURNAL OF MATHEMATICS}

\section{EDITORS}

H. SAMELSON

Stanford University

Stanford, California 94305

\section{RichaRd PIERCe}

University of Washington

Seattle, Washington 98105
J. DUGUNDJI

Department of Mathematics

University of Southern California

Los Angeles, California 90007

RICHARD ARENS

University of California

Los Angeles, California 90024

\section{ASSOCIATE EDITORS}

E. F. BECKENBACH

B. H. NeUManN

F. WOLF

K. YosHIDA

\section{SUPPORTING INSTITUTIONS}

UNIVERSITY OF BRITISH COLUMBIA CALIFORNIA INSTITUTE OF TECHNOLOGY UNIVERSITY OF CALIFORNIA MONTANA STATE UNIVERSITY

UNIVERSITY OF NEVADA

NEW MEXICO STATE UNIVERSITY

OREGON STATE UNIVERSITY

UNIVERSITY OF OREGON

OSAKA UNIVERSITY

UNIVERSITY OF SOUTHERN CALIFORNIA
STANFORD UNIVERSITY

UNIVERSITY OF TOKYO

UNIVERSITY OF UTAH

WASHINGTON STATE UNIVERSITY

UNIVERSITY OF WASHINGTON

${ }^{*} \quad{ }^{*} \quad{ }^{*}$
AMERICAN MATHEMATICAL SOCIETY
CHEVRON RESEARCH CORPORATION
TRW SYSTEMS
NAVAL WEAPONS CENTER




\section{Pacific Journal of Mathematics}

\section{Vol. 33, No. $1 \quad$ March, 1970}

Mir Maswood Ali, On some extremal simplexes ................... 1

Silvio Aurora, On normed rings with monotone multiplication........... 15

Silvio Aurora, Normed fields which extend normed rings of integers....... 21

John Kelly Beem, Indefinite Minkowski spaces..................... 29

T. F. Bridgland, Trajectory integrals of set valued functions ........... 43

Robert Jay Buck, A generalized Hausdorff dimension for functions and sets ......................................... 69

Vlastimil B. Dlab, A characterization of perfect rings . . . . . . . . . . . . 79

Edward Richard Fadell, Some examples in fixed point theory ............ 89

Michael Benton Freeman, Tangential Cauchy-Riemann equations and uniform approximation ............................. 101

Barry J. Gardner, Torsion classes and pure subgroups ................ 109

Vinod B. Goyal, Bounds for the solution of a certain class of nonlinear

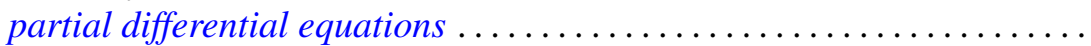

Fu Cheng Hsiang, On C, 1 summability factors of Fourier series at a given

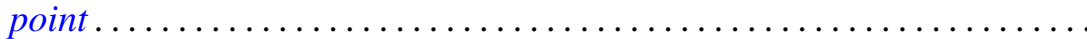

Lawrence Stanislaus Husch, Jr., Homotopy groups of PL-embedding

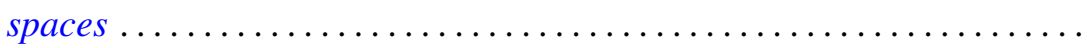

Daniel Ralph Lewis, Integration with respect to vector measures..........

Marion-Josephine Lim, $\mathscr{L}-2$ subspaces of Grassmann product spaces

Stephen J. Pierce, Orthogonal groups of positive definite multilinear functionals

W. J. Pugh and S. M. Shah, On the growth of entire functions of bounded index.

Siddani Bhaskara Rao and Ayyagari Ramachandra Rao, Existence of triconnected graphs with prescribed degrees . . .

Ralph Tyrrell Rockafellar, On the maximal monotonicity of subdifferential

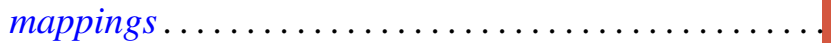

R. Shantaram, Convergence of a sequence of transformations of distribution functions. II ...............................

Julianne Souchek, Rings of analytic functions..............

Ted Joe Suffridge, The principle of subordination applied to functions of several variables...

Wei-lung Ting, On secondary characteristic classes in cobordism

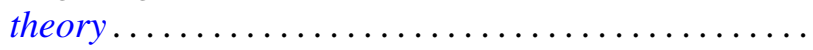

Pak-Ken Wong, Continuous complementors on $B^{*}$-algebras ...

Miyuki Yamada, On a regular semigroup in which the idempotents form a band. 